\title{
Sistemas de Comunicação na Organização Hospitalar
}

\author{
Cristiano Sena da Conceição ${ }^{1}$, Francisco Antônio Pereira Fialho ${ }^{2}$, João Bosco da Mota \\ Alves $^{3}$, Luiz Alberto Gomes ${ }^{4}$, Rosângela Martins Gueudeville ${ }^{5}$ \\ Programa de Engenharia e Gestão do Conhecimento - UFSC \\ 1 cristianosena@cmbox1.ufsc.br \\ ${ }^{2}$ fapfialho@egc.ufsc.br \\ 3jbosco@egc.ufsc.br \\ 4luiz@ecv.ufsc.br \\ ${ }^{5}$ rosangela@egc.ufsc.br
}

\begin{abstract}
Resumo
Este artigo tem por objetivos apresentar a organização hospitalar como um sistema complexo sob a luz da Teoria Geral de Sistemas em busca do entendimento das dificuldades de comunicação entre seus subsistemas e propor mediante a atual capacidade computacional das ontologias em saúde, a telemática como uma ferramenta baseada nas tendências das novas Tecnologias de Informação e Comunicação, em busca de uma comunicação eficaz entre os profissionais de saúde, pacientes e organização hospitalar. Palavras-chave: Complexidade, sistemas de comunicação, hospital e telemática.
\end{abstract}

\begin{abstract}
The aims of this paper are present the hospitalar organization how a complex system on the light of the General System Theory looking for attendment of the communication difficults between your system and propose by means of the actual computacionaly capacity of the ontologies in health, the telematics like a tool based in tendency of the news technologies of information and communication looking for eficacy communication between healthcare professionals, patients and hospitalar organization.

Key-words: Complexity, communication of systems, hospital e telematics.
\end{abstract}

\section{Introdução}

A prática clínica dos profissionais de saúde no ambiente hospitalar é caracterizada por uma grande e complexa rede de relacionamentos, na qual muitas informações tramitam pelos diversos subsistemas da organização hospitalar.

O sucesso da prestação de serviço na saúde é dependente da qualidade e veracidade das informações, assim falhas nos sistemas de comunicação afetam negativamente o processo de transmissão de informações e geração do conhecimento entre os participantes dos subsistemas hospitalares. Em geral, a organização hospitalar enquanto um sistema é composto dos subsistemas descritos a seguir:

- Administrativo representado pela diretoria e os setores financeiros;

- Serviços gerais que incluem recepção, higienização, dentre outros;

- Unidades de internamento divididas em emergência, enfermarias, apartamentos, unidades de terapia intensiva, semi-intensiva e centro cirúrgico;

- Clientes (pacientes e familiares).

Todos estes componentes são constituídos de agentes que interagem entre si e entre suas unidades, com relações de dependência e influência, em uma dinâmica constante e não linear, caracterizando um sistema complexo, pois como afirma Axelrod [1] "complexidade" não denota simplesmente "muitas partes em movimento". Ao contrário, indica que o sistema consiste de partes as quais interagem, assim o sistema é conseqüente da organização de seus componentes e influenciado fortemente por eles. A complexidade normalmente resulta das características que são propriedades do sistema que as partes separadas não têm. 
Um atendimento integral requer constante troca de dados e informações por todas as pessoas envolvidas no processo terapêutico: profissionais, pacientes e gestores. Assim, neste sistema ocorrem diversos fatos que são filtrados por pessoas ou programas (softwares) tornando-se dados, os quais dotados de significado passam a ser informação, que quando apropriada por cada membro do sistema é chamada de conhecimento.

As informações presentes no sistema e subsistemas da organização hospitalar devem ser agregadas ao conhecimento individual de cada membro do sistema e não ficar restrita, para tanto é necessário uma padronização na comunicação facilitando a gestão do conhecimento dentro da organização hospitalar.

Desta forma, no segundo item deste artigo serão discutidos os problemas de comunicação e as formas de padronização da comunicação no ambiente hospitalar, para no terceiro item sugerir e demonstrar a telemática como uma tendência das novas Tecnologias de Informação e Comunicação que pode auxiliar a comunicação no sistema hospitalar.

\section{Comunicação no Sistema Hospitalar}

A comunicação é um processo complexo envolvendo duas ou mais pessoas, no qual são trocadas informações, podendo ser diagnosticadas e solucionadas dificuldades. Silva [16] define a comunicação como um ato criativo, no qual existe um agente emissor, um receptor e há uma troca entre as pessoas, formando um sistema de interação e reação, ou seja, um processo recíproco, que provoca a curto ou longo prazo, mudanças na forma de sentir, pensar e atuar dos envolvidos.

Uma organização hospitalar compreende uma instituição de médio ou grande porte que presta serviços de assistência à saúde, envolvendo diversos profissionais da área de saúde, além dos gestores, clientes e equipe de serviços gerais, formando uma rede de relacionamentos. Quanto mais complexa é a rede de relacionamento da organização hospitalar, maior a possibilidade de falha na comunicação, fato que predispõe ao "erro" na prática do profissional de saúde. Esta falha pode repercutir na má qualidade da prestação de saúde bem como em maiores gastos pertinentes ao prolongamento da permanência do cliente no hospital. Um estudo realizado por Trummer et al [19] demonstrou que a melhora na comunicação entre profissionais de saúde e seus pacientes repercutem efetivamente nas intervenções hospitalares.

Britten et al [5] também realizou um estudo sobre comunicação na área de saúde, no qual foram relatadas 14 categorias de desentendimentos de comunicação entre profissionais de saúde e seus pacientes que envolvem fatores como: não conhecimento e distância entre os agentes, informações conflitantes, discordância sobre atribuição de responsabilidades, falência da comunicação sobre as decisões tomadas e dos fatores ligados ao relacionamento.

A falta de padronização na comunicação entre os subsistemas de um hospital é reconhecida como uma das grandes causas do insucesso na comunicação entre os profissionais de saúde e a organização. O resultado é a deficiência na qualidade da informação, definida como perda de seu valor e relevância, assim como predispõe a ambigüidades e dificuldades na troca de informações entre os subsistemas e inter-sistemas organizacionais de saúde.

Um dos principais métodos para consecução da padronização na comunicação entre profissionais de saúde é a estruturação na composição dos relatórios ou dos prontuários. Uma das formas mais utilizadas é o modelo estruturado de preenchimento das informações de saúde. Este deve reduzir o uso de textos livres normalmente escritos à mão e aumentar o uso de códigos específicos que contenham um significado específico para que assim seja possível a sua digitalização. [10, 20]

Desta forma a busca pela codificação das terminologias em saúde é o ponto chave para o início de uma padronização da comunicação. Para tal, os pesquisadores se apoiam em uma importante área do conhecimento chamada: ontolologia.

Segundo a enciclopédia virtual Wikipedia [23] o termo ontologia vem do grego: ta onta+logoi: "conhecimento do ser". Este conceito tem sido retomado devido ao estudo das redes semânticas. Conforme Steiner et al [18] as ontologias servem como guias de extração e integração de informações relevantes, normalmente pertencentes a algum domínio mesmo que oriundas de fontes diversas.

As ontologias ligadas à área de saúde baseadas em uma semântica única proporcionam a codificação da terminologia em saúde estabelecendo uma linguagem comum. Como exemplos de ontologias temos: a CID-10 (Classificação Estatística Internacional de Doenças e Problemas Relacionados à Saúde, $10^{\mathrm{a}}$ Revisão) que fornece um modelo baseado na etiologia, anatomia e causas externas das lesões, a CIF (Classificação Internacional da Funcionalidade, Incapacidade e Saúde) e a SNOMED (Systemized Nomenclature of Medicine Clinical Terms (SNOMED CT ${ }^{\mathbb{B}}$ ) [17], uma das maiores bases de dados, com termos de saúde que em julho 2005 continha cerca de 366,170 conceitos associados a mais de 993,420 termos e 1,46 milhões de relações semânticas. No Brasil existe o DeCS (Descritores em Saúde) ligado à bireme desenvolvido a partir do MeSH Medical Subject Headings da "U.S. National Library of Medicine" que contem cerca de 26851 descritores. [3, $13,17,22]$.

O surgimento das ontologias na área de saúde, através da utilização dos códigos, foi o passo inicial para a digitalização dos dados relativos à prática de seus profissionais. O emprego de termos e seus respectivos códigos organizados em uma estrutura conceitual resulta em um sistema de classificação que provê fundamentos para o desenvolvimento de muitos softwares. Um dos primeiros exemplos de softwares foi a criação dos sistemas de prontuário médico eletrônicos [18]. 
As possibilidades computacionais permitidas pela padronização das terminologias em saúde associada à evolução das Tecnologias de Informação e Comunicação (TIC's) têm estimulado o interesse da comunidade científica por modelos que explorem a comunicação entres os subsistemas da organização hospitalar. Isto porque, a eficácia da comunicação entre profissionais reduz a chance de erros de interpretação, reduz os dias de internamento, estimula a prática baseada em evidências e melhora a coordenação entre as atividades dos profissionais de saúde e sua organização. $[6,11]$.

Assim, métodos que procurem criar um ambiente favorável à comunicação dentro da organização hospitalar devem permitir um aumento na velocidade de compreensão e troca dos dados e reduzir a obsolescência das informações em saúde.

\section{Introdução das TIC's na organização hospitalar.}

A tendência para prática de saúde é a integração das informações que de uma maneira geral é inerente à expansão das TIC's dentro das organizações hospitalares. Estas tecnologias permitem criar um ambiente eletrônico que suportam a padronização das terminologias e intercâmbio de informações. O resultado é a melhora na comunicação entre os subsistemas de uma organização hospitalar [4, 9].

A associação da Medicina com as Tecnologias de Informação e Comunicação resultou no surgimento da Telemedicina, uma forma de se prestar assistência médica, treinamento, informações e imagens para profissionais e pacientes, utilizando recursos tecnológicos, como videoconferência, softwares, internet, dentre outros.

A Organização Mundial de Saúde [WHO, 21] define Telemedicina como: "a oferta de serviços ligados aos cuidados com a saúde, nos casos em que a distância é um fator crítico; tais serviços são prestados por profissionais da área da saúde, usando tecnologias de informação e de comunicação para o intercâmbio de informações válidas para diagnósticos, prevenção e tratamento de doenças e a contínua educação de prestadores de serviços em saúde, assim como para fins de pesquisas e avaliações".

A utilização dos portais de telemedicina vem crescendo para minimizar os problemas de diferenças de tratamento de saúde entre regiões de um país ou até mesmo entre países, pois possibilita que centros de excelência ofereçam seus serviços e conhecimentos à distância. Entre os benefícios que a telemedicina oferece atualmente, podem-se destacar melhores condições de organização de dados médicos, facilidade de acesso à informação e treinamento de profissionais, através da troca de informações e educação à distância [2].

Segundo Packer [14] os portais têm se mostrado como uma excelente ferramenta para construção e disseminação do conhecimento, o que é extremamente importante para o desenvolvimento da ciência e prática da saúde.

Um dos grandes avanços das Tecnologias de Informação e Comunicação aplicado na área de saúde é a telemática, que surge através da convergência da Engenharia Biomédica, Informática e Telecomunicações. A telemática não está orientada para os aspectos clínicos como a Telemedicina, mas para a utilização dos serviços de saúde à distância a fím de promover a saúde e o controle de doenças, através da instrução da equipe médica, dos pacientes e comunidades.

Os benefícios da Telemática são: melhora da comunicação entre os profissionais, pacientes e a organização de saúde, melhora a eficácia da coordenação das atividades inter-profissionais, além de provê uma base de dados eletrônicos que permitirá a qualquer profissional ao redor do mundo ter acesso às informações sobre a saúde de seu paciente, transpondo barreiras geográficas, culturais e socioeconômicas. Por serem dados eletrônicos também permitem o armazenamento das informações da saúde do paciente ao longo de sua vida [6].

Alguns fatores dificultam a implementação da Telemática, como a escolha correta da tecnologia a ser empregada, pois é necessário a utilização de programas que maximizem a automação de aquisição de dados, organizem e disseminem as informações entre os setores para que possam ser convertidas em conhecimento; convencer e motivar os usuários para obter sucesso na implementação; manter a segurança, privacidade e atualização dos dados.

Existem algumas experiências sobre o uso da telemática na saúde, mas é evidente que não é aplicada em larga escala bem como não há generalização da digitalização das informações da área de saúde. [8]

No Brasil existem algumas experiências que tratem de melhorias na troca de informações entre as organizações de saúde. Um exemplo é o portal de Telemedicina do estado de Santa Catarina, onde estão disponíveis os prontuários eletrônicos dos pacientes atendidos em alguns municípios, em especial no Hospital Universitário Polydoro Ernani de São Thiago. Neste portal os profissionais de saúde podem acessar de forma rápida e segura todas as informações de saúde relativas ao histórico do paciente.

Outros exemplos de instituições que estão utilizando a Telemedicina são: Faculdade de Medicina da Universidade de São Paulo atuando na criação e desenvolvimento de sistemas interface Web, TeleEducação e Tele-Diagnóstico, como o Web site para capacitar pessoas a identificar lesões malignas de pele [12] e o Setor de Telemedicina da Universidade Federal de São Paulo (UNIFESP) que criou o programa ATENAS, o qual através de recursos mediados pelo computador integra os profissionais de saúde, agentes comunitários e a comunidade aos serviços de saúde localizados no Hospital da UNIFESP [17].

$\mathrm{O}$ uso das novas tecnologias de informação e comunicação na área de saúde está iniciando um caminho de grandes possibilidades que cresce na mesma velocidade de avanço das inovações tecnológicas. 
Porém, ainda são poucas as experiências e os exíguos modelos de gestão do conhecimento têm mostrado fraca eficiência. Isto se deve a baixa qualificação dos profissionais usuários e reduzidos investimentos em gestão e inovação [7].

\section{Conclusão}

A comunicação entre os subsistemas de uma organização hospitalar deve ser vista como um sistema dinâmico e complexo, no qual, as partes interagem com o todo. Desta forma, os subsistemas devem falar uma linguagem comum e estimular a participação de todos os agentes. Neste âmbito, a padronização das terminologias em saúde através de códigos tem oferecido uma base sólida para o desenvolvimento de aplicativos computacionais como, por exemplo, o prontuário eletrônico e assim aumentar a velocidade de troca, diminuir a obsolescência, ambigüidades e manter a relevância da informação.

As novas Tecnologias de Informação e Comunicação oferecem um amplo suporte à melhora da eficácia da comunicação entre os profisssionais de saúde e a organização hospitalar. A telemática pode ser utilizada como uma ferramenta de comunicação entre os sistemas e subsistemas da organização hospitalar, ao prover maior portabilidade das informações e acesso rápido a uma base de dados confiáveis, seguras e atualizadas, o que repercute não somente na comunicação, integração e no conhecimento dos profissionais, mas também na assistência prestada aos pacientes. A informações podem também ser utilizadas por outras instituições hospitalares disponibilizando recursos para outras comunidades desprovidas de acesso ou trocando informações com instituições com perfil semelhante.

Porém, a busca por um modelo que contemple a participação global dos agentes de uma organização encontra estímulos e barreiras, pois, de um lado os pacientes se mostram mais pró-ativos e questionam sobre transparência, melhora qualidade das informações, cooperação e atualização terapêutica entre os profissionais, do outro, os profissionais de saúde ainda se mostram reticentes aos avanços proporcionados pelas novas tecnologias de informação e comunicação.

Assim, frente aos obstáculos da não integração de todos os agentes do sistema hospitalar, se faz necessário novos estudos que ampliem as possibilidades da telemática para uso mais sistêmico, enfocando principalmente a criação de mecanismos para estimular o uso das novas Tecnologias de Informação e Comunicação por parte dos profissionais de saúde.

\section{Referências}

[1].AXELROD, R.M. The complexity of cooperation : agent-bases models models of competition and collaboration. New Jersey: Princeton University Press, 1997.

[2].BEUSCART, R. et al. Telecommunication in health care for a better coordination between hospitals and GP's: routine application of the "IsarTelematics" project. IEEE Transactions on Information Techonology Biomedicine, vol. 3, n. 2, Junho, 1999.

[3].[BIREME]. Texto retirado do site < http://decs.bvs.br/P/decswebp.htm $>$ com acesso em 21/05/06.

[4]. BRANGER P. et al. Problems in Communication between General Practitioners and Internal Medicine Consultants. International Journal of Medical Informatics, vol. 20, 1995.

[5].BRITTEN, N. et al. Misunderstandings in prescribiging decisions in general practice: qualitative study. BMJ vol. 320, 2000.

[6]. BRUNETAUD, J. M., BEUSCART, R. J., ALAO, O. O. Health Telematics: a challenge for healthcare quality. Proceedings of the $23^{\text {rd }}$ Annual EBMS International Conference. October, 2001.

[7].COSTA, S.M.S. Mudanças no processo de comunicação científica: o impacto do uso de novas tecnologias. In: MUELLER, S.P.M.; PASSOS, E.J.L., (Org.). Comunicação científica. Brasília: DCI/UnB, 2000.

[8].GRIGSBY, J., SANDERS, J. H. Where it is and where it's going. Annals of Internal Medicine, 129, 1998.

[9].HAMPSON, J., ROBERTS, R., MORGAN, D. Shared Care: A Review of the Literature. Family Practice, vol. 13, no. 3, 1996

[10].HARRIS, R. M., RUGGIERI, A. P. CHUTE, C.G. From Clinical Records to Regulatory Reporting: Formal Terminologies as Foundation. Health care financing review. Vol. 24, Number 3, 2003.

[11].HOLLE, R., ZAHLMANN G., Evaluation of telemedical service. IEEE. Transacations on Information Technology in Biomedicine 3, 1999.

[12].Informática Médica da Faculdade de Medicina da Universidade de São Paulo - HCFMUSP. Texto $\mathrm{re} \mathrm{ti} \mathrm{rad} \mathrm{o} \mathrm{d} \mathrm{o} \mathrm{s} \mathrm{i} \mathrm{t} \mathrm{e}$ $<$ http://www.netsim.fm.usp.br/telemedicina/ $>$ com acesso em 29/10/2006.

[13].MAYO N.E., POISSANT L., AHMED S., FINCH, L., HIGGINS J., SALBACH, N. M., SOICHER, J., JAGLAL, S. J. Incorporating the Internacional Classification of Functioning, Disability, and Health (ICF) into an Eletronic Health Record to Creat Indicators of Function: Proof of Concept Using the SF-12. Am Med Inform Assoc. Volume 11 Number 6 Nov / Dec 2004.

[14].PACKER, A. A Biblioteca Virtual em Saúde como espaço público de gestão em rede e acesso eqüitativo às fontes de informação e conhecimento científico, técnico e factual. Belo Horizonte: VI Congresso Nacional da Rede Unida, 2005.

[15].Setor de Telemedicina (SET-DIS). Texto retirado do site $<$ http://www.unifesp.br/dis/set> com acesso em 15/10/2006. 
[16].SILVA, M. J. P. Comunicação tem remédio: a comunicação nas relações interpessoais em saúde. São Paulo: Gente, 1996.

[17].[SNOMED CT]. Texto retirado do site $<$ http://www.snomed.org/snomedct/index.html $>$ com acesso em 21/05/06.

[18].STEINER, A. W. RYSER, L., HUBER E., UEBELHART D., AESCHILIMANN A., STUCKI G. Use of the ICF Model as a Clinical ProblemSolving Tool in Physical Therapy and Rehabilitation Medicine. Physical Therapy, vol. 82, n. 11, November 2002.

[19].TRUMMER, F. U. et al. Does physician-patient communication that aims at empowering patients improve clinical outcome? A case study. Patient Education and Counseling 61 (2006).

[20].UECKERT, F., GOERZ, M., ATAIAN, M., TESSMANN, S. PROCKOSCH, H. U.
Empowerment of patients and communication with health care professionals through an electronic health record. International Journal of Medical Informatics, 2003.

[21]. [WHO]. World Health Organization. Texto retirado do site $<$ http://www.who.orgt $>$ com acesso em 9/10/2006.

[22].[WHO]. World Health Organization. International Classification of Functioning Disability and Health: ICF. Geneva: WHO; 2002.

[23].[WIKIPEDIA]. Texto retirado do site $<$ http://pt.wiktionary.org/wiki/Ontologia> com acesso em 22/05/06. 